\title{
Estimation of High Resolution Images and Registration Parameters from Low Resolution Observations ${ }^{\star}$
}

\author{
Salvador Villena ${ }^{1}$, Javier Abad ${ }^{2}$, Rafael Molina ${ }^{2}$, and Aggelos K. Katsaggelos ${ }^{3}$ \\ 1 Dpto. de Lenguajes y Sistemas Informáticos, Universidad de Granada, \\ 18071 Granada, Spain \\ svillena@ugr.es \\ 2 Dpto. de Ciencias de la Computación e I.A., Universidad de Granada, \\ 18071 Granada, Spain \\ \{abad,rms\}@decsai.ugr.es \\ 3 Dept. of Electrical and Computer Engineering, Northwestern University, \\ Evanston, IL 60208-3118 \\ aggk@ece.nwu .edu
}

\begin{abstract}
In this paper we consider the problem of reconstructing a high resolution image from a set of undersampled and degraded frames, all of them obtained from high resolution images with unknown shifting displacements between them. We derive an iterative method to estimate the unknown shifts and the high resolution image given the low resolution observations. Finally, the proposed method is tested on real images.
\end{abstract}

\section{Introduction}

High resolution images can, in some cases, be obtained directly from high precision optics and charge coupled devices (CCDs). However, due to hardware and cost limitations, imaging systems often provide us with only multiple low resolution images. In addition, there is a lower limit as to how small each CCD can be, due to the presence of shot noise [1] and the fact that the associated signal to noise ratio (SNR) is proportional to the size of the detector [8].

Over the last two decades research has been devoted to the problem of reconstructing a high resolution image from multiple shifted undersampled, and degraded frames (see [4] for a recent review). A key part of the high resolution reconstruction problem is the estimation of the shifts between the images.

Most of the reported approaches on shift estimation for super-resolution, first estimate the displacement vectors either by interpolating the low resolution observations and then finding the registration parameters or by finding the low resolution registration parameters in the low resolution domain and then interpolating them (consider [4] again), where the high resolution image and the registration parameters are estimated simultaneously, can be found in $[10,9,12$, $2]$.

* This work has been partially supported by the "Comisión Nacional de Ciencia y Tecnología" under contract TIC2003-00880. 
In this paper, starting from the low to high resolution method described in [6, 11], we propose a new iterative method to alternatively estimate the registration parameters and to obtain a high resolution image from a set of low resolution observations.

The paper is organized as follows. The problem formulation is described in section 2. The process to estimate the displacements between the images is described in section 3. Once the registration parameters have been obtained the application of the Bayesian paradigm to calculate the MAP high resolution image and estimate the regularization hyperparameters is described in section 4 . The iterative estimation of the registration parameters and the high resolution image is discussed in section 5. Experimental results are described in section 6 . Finally, section 7 concludes the paper.

\section{Problem Formulation}

Consider a set $\mathbf{g}=\left\{\mathbf{g}_{1}, \ldots, \mathbf{g}_{Q}\right\}$ of $Q \geq 1$ low resolution images with $N_{1} \times N_{2}$ pixels. The low resolution sensors obtaining the low resolution images are shifted with unknown horizontal and vertical displacements with respect to each other by values proportional to $\left(T_{1} / L\right) \times\left(T_{2} / L\right)$, where $T_{1} \times T_{2}$ is the size of each low resolution sensing element and $L$ denotes the high resolution magnification factor. Our aim is to reconstruct an $M_{1} \times M_{2}$ high resolution image, f, with $M_{1}=L \times N_{1}$ and $M_{2}=L \times N_{2}$, from the set of low resolution observations.

We now relate the low resolution observed images in $\mathrm{g}$ to the unknown high resolution image $\mathbf{f}$ we want to estimate. Since the low resolution sensors obtaining the low resolution images are shifted with unknown horizontal and vertical displacements with respect to each other by values proportional to $T_{1} / L \times T_{2} / L$ we have that each $\mathbf{g}_{i}, i=1, \ldots, Q$ results from $\mathbf{f}$ through motion compensation, filtering, and subsampling:

$$
\mathbf{g}_{\mathbf{q}}=\mathbf{A H C}\left(\mathbf{d}_{\mathbf{q}}\right) \mathbf{f}+\epsilon_{\mathbf{q}} \quad \mathbf{q}=1, \ldots, Q,
$$

where $\mathbf{C}\left(\mathbf{d}_{\mathbf{q}}\right)$ is the $\left(M_{1} \times M_{2}\right) \times\left(M_{1} \times M_{2}\right)$ matrix defined by $\mathbf{C}\left(\mathbf{d}_{\mathbf{q}}\right) \mathbf{f}(a, b)=$ $\left.\mathbf{f}\left(a+\mathbf{d}_{\mathbf{q}}^{x}, b+\mathbf{d}_{\mathbf{q}}^{y}\right)\right)$, where $\mathbf{d}_{\mathbf{q}}=\left(\mathbf{d}_{\mathbf{q}}^{x}, \mathbf{d}_{\mathbf{q}}^{y}\right)$ denotes horizontal and vertical displacements, matrix $\mathbf{H}$ of size $\left(N_{1} \times N_{2}\right) \times\left(L N_{1} \times L N_{2}\right)$ describes a high resolution filtering operation, $\mathbf{A}$ is the downsampling matrix with size $N_{1} N_{2} \times\left(M_{1} M_{2}\right)$ and $\epsilon_{\mathbf{q}}$ a random independent noise with variance $\beta_{\mathbf{q}}^{-1}$.

Matrices $\mathbf{A}$ and $\mathbf{H}$ are assumed to be known. $\mathbf{A H}$ models optical distortion together with CCD pixel resolution [11]. The matrix $\mathbf{H}$ is assumed to be blockcirculant and $\mathbf{C}\left(\mathbf{d}_{\mathbf{q}}\right)$ will be approximated by a block-circulant matrix as well.

Our problem can now be formulated as the estimation of the set of displacements, $\mathbf{d}=\left\{\mathbf{d}_{\mathbf{1}}, \ldots, \mathbf{d}_{\mathbf{q}}\right\}$, and the high resolution image $\mathbf{f}$. In order to perform this task we will proceed in two steps; first we will estimate the displacements $\mathbf{d}$ and then the high resolution image $\mathbf{f}$. 


\section{Estimation of the Displacement Vectors}

To obtain the high resolution image $\mathbf{f}$, we need to accurate estimate the pixel displacement $\mathbf{d}_{\mathbf{q}}$ in (1). In this paper we estimate the registration parameters as follows. From (1), we first consider for each $\mathbf{q} \in\{1, \ldots, Q\}$, the following low to high resolution observation model with just one observation,

$$
\mathbf{g}_{\mathbf{q}}=\mathbf{A H u}_{\mathbf{q}}+\psi_{\mathbf{q}} \quad \mathbf{q}=1, \ldots, Q,
$$

where $\mathbf{u}_{\mathbf{q}}$ is an $M_{1} \times M_{2}$ high resolution image, $\mathbf{H}$ and $\mathbf{A}$ have been defined in (1) and $\psi_{\mathbf{q}}$ is random independent noise with variance $\mu_{\mathbf{q}}^{-1}$.

We then use as prior model for $\mathbf{u}_{\mathbf{q}}$ a simultaneous autoregression (SAR) [7], that is,

$$
p\left(\mathbf{u}_{\mathbf{q}} \mid \alpha_{\mathbf{q}}\right)=\frac{1}{Z_{\text {prior }}\left(\alpha_{\mathbf{q}}\right)} \exp \left\{-\frac{1}{2} \alpha_{\mathbf{q}}\left\|\mathbf{C} \mathbf{u}_{\mathbf{q}}\right\|^{2}\right\},
$$

where the parameter $\alpha_{\mathbf{q}}$ measures the smoothness of the 'true' image, $Z_{\text {prior }}\left(\alpha_{\mathbf{q}}\right)$ $=\left(\prod_{i, j} \lambda_{i j}^{2}\right)^{-1 / 2}\left(2 \pi / \alpha_{\mathbf{q}}\right)^{\left(M_{1} \times M_{2}\right) / 2}$ and $\lambda_{i j}=1-2 \phi\left(\cos \left(2 \pi i / M_{1}\right)+\cos \left(2 \pi j / M_{2}\right)\right)$, $i=1,2, \ldots, M_{1}, j=1,2, \ldots, M_{2}$ and $\mathbf{C}$ is the Laplacian.

Then we select as estimate of $\mathbf{u}_{\mathbf{q}}$,

$$
\hat{\mathbf{u}}_{\mathbf{q}}=\arg \min _{\mathbf{u}_{\mathbf{q}}}\left\{\alpha_{\mathbf{q}}\left\|\mathbf{C} \mathbf{u}_{\mathbf{q}}\right\|^{2}+\mu_{\mathbf{q}}\left\|\mathbf{g}_{\mathbf{q}}-\mathbf{A} \mathbf{H} \mathbf{u}_{\mathbf{q}}\right\|^{2}\right\}
$$

The high resolution estimate $\hat{\mathbf{u}}_{\mathbf{q}}$ is found together with estimates of $\alpha_{\mathbf{q}}$ and $\mu_{\mathbf{q}}$ using the method described in $[6,11,5]$.

One of the $\hat{\mathbf{u}}_{\mathbf{q}}$ 's (call it $\hat{\mathbf{u}}$ ) is an initial estimate of $\mathbf{f}$, and we proceed with the estimation of the registration parameters $\mathbf{d}=\left\{\mathbf{d}_{\mathbf{1}}, \ldots, \mathbf{d}_{\mathbf{q}}\right\}$. In this paper we have used the following two approaches.

\subsection{Method I: Global Correlation}

Given $\hat{\mathbf{u}}_{\mathbf{q}}$ and the initial estimate, $\hat{\mathbf{u}}$, of the high resolution image $\mathbf{f}$ we calculate the registration parameters between $\hat{\mathbf{u}}_{\mathbf{q}}$ and $\hat{\mathbf{u}}$ by finding

$$
\left(\hat{\mathbf{d}}_{\mathbf{q}}^{x}, \hat{\mathbf{d}}_{\mathbf{q}}^{y}\right)=\arg \max _{\mathbf{d}_{\mathbf{q}}^{x}, \mathbf{d}_{\mathbf{q}}^{y}} C_{\hat{\mathbf{u}}_{\mathbf{q}}, \hat{\mathbf{u}}}\left(\mathbf{d}_{\mathbf{q}}^{x}, \mathbf{d}_{\mathbf{q}}^{y}\right)
$$

where

$$
C_{\hat{\mathbf{u}}_{\mathbf{q}}, \hat{\mathbf{u}}}\left(\mathbf{d}_{\mathbf{q}}^{x}, \mathbf{d}_{\mathbf{q}}^{y}\right)=\sum_{n_{1}} \sum_{n_{2}} \hat{\mathbf{u}}_{\mathbf{q}}\left(n_{1}, n_{2}\right) \hat{\mathbf{u}}\left(n_{1}+\mathbf{d}_{\mathbf{q}}^{x}, n_{2}+\mathbf{d}_{\mathbf{q}}^{y}\right)
$$

\subsection{Method II: Local Correlation}

Given $\hat{\mathbf{u}}_{\mathbf{q}}$ and $\hat{\mathbf{u}}$ we compute a set of points of interest in image $\hat{\mathbf{u}}_{\mathbf{q}}$ (see for instance [3]). Then, for each pixel location $\left(n_{x}, n_{y}\right)$, corresponding to a point of interest, consider a block of small size centered around it and search for the location of the best-matching block of the same size in a limited area around pixel $\left(n_{x}, n_{y}\right)$ in image $\hat{\mathbf{u}}$. 
Let $\mathbf{d}_{\mathbf{q}}(i)$ for $i=1, \ldots$, points of interest be the set of obtained registration parameters; we then create the list $L=\left\{\mathbf{d}_{\mathbf{q}}^{n}, n=1, \ldots, N\right\}$, of the $\mathrm{N}$ most frequently appearing values of the registration parameters ( $\mathrm{N}$ should cover a high percentage of the points of interest). For each $\mathbf{d}_{\mathbf{q}}^{n}$ find the correlation between the central part of $\hat{\mathbf{u}}_{\mathbf{q}}$ of size $M_{1} / 2 \times M_{2} / 2$ and the same area in image $\hat{\mathbf{u}}$ displaced by $\mathbf{d}_{\mathbf{q}}^{n}$. Select the registration parameter maximizing this correlation.

\section{Bayesian Analysis}

Once we know the registration vector $\mathbf{d}$, from (1), the probability density function of $\mathbf{g}_{\mathbf{q}}$, with $\mathbf{f}$ the 'true' high resolution image, is given by

$$
p\left(\mathbf{g}_{\mathbf{q}} \mid \mathbf{f}, \beta_{\mathbf{q}}\right)=\frac{1}{Z\left(\beta_{\mathbf{q}}\right)} \exp \left[-\frac{\beta_{\mathbf{q}}}{2}\left\|\mathbf{g}_{\mathbf{q}}-\mathbf{W}_{\mathbf{q}} \mathbf{f}\right\|^{2}\right]
$$

where $Z\left(\beta_{\mathbf{q}}\right)=\left(2 \pi / \beta_{\mathbf{q}}\right)^{\left(N_{1} \times N_{2}\right) / 2}, \beta_{\mathbf{q}}$ is the inverse of the noise variance and $\mathbf{W}_{\mathbf{q}}=\mathbf{A H C}\left(\mathbf{d}_{\mathbf{q}}\right)$.

Since we have multiple low resolution images, the probability density function of $\mathbf{g}$ given $\mathbf{f}$ is

$$
\begin{aligned}
& p(\mathbf{g} \mid \mathbf{f}, \underline{\beta})=\prod_{\mathbf{q}} p\left(\mathbf{g}_{\mathbf{q}} \mid \mathbf{f}, \beta_{\mathbf{q}}\right) \\
& =\frac{1}{Z_{\text {noise }(\underline{\beta})}} \exp \left[-\frac{1}{2} \sum_{\mathbf{q}} \beta_{\mathbf{q}}\left\|\mathbf{g}_{\mathbf{q}}-\mathbf{W}_{\mathbf{q}} \mathbf{f}\right\|^{2}\right]
\end{aligned}
$$

where $\underline{\beta}=\left(\beta_{1}, \ldots, \beta_{Q}\right)$ and $Z_{\text {noise }}(\underline{\beta})=\prod_{\mathbf{q}} Z\left(\beta_{\mathbf{q}}\right)$.

As prior model for $\mathbf{f}$ we use the simultaneous autoregression (SAR) defined in $(3)$, that is

$$
p(\mathbf{f} \mid \alpha)=\frac{1}{Z_{\text {prior }}(\alpha)} \exp \left\{-\frac{1}{2} \alpha\|\mathbf{C} \mathbf{f}\|^{2}\right\},
$$

Note that this SAR model is equivalent to the use of the Laplacian operator to regularize the high resolution image estimate.

The Bayesian analysis is performed to estimate the hyperparameters, $\alpha$ and $\underline{\beta}$, and the high resolution image. In this paper we use the following two steps:

\section{Step I: Estimation of the Hyperparameters}

$\hat{\alpha}$ and $\underline{\hat{\beta}}$ are first selected as

$$
\hat{\alpha}, \underline{\hat{\beta}}=\arg \max _{\alpha, \underline{\beta}} \mathcal{L}_{\mathbf{g}}(\alpha, \underline{\beta})=\arg \max _{\alpha, \underline{\beta}} \log p(\mathbf{g} \mid \alpha, \underline{\beta}),
$$

where $p(\mathbf{g} \mid \alpha, \underline{\beta})=\int_{\mathbf{f}} p(\mathbf{f} \mid \alpha) p(\mathbf{g} \mid \mathbf{f}, \underline{\beta}) d \mathbf{f}$.

The solution to this equation is obtained with the EM-algorithm with $\mathcal{X}^{t}=$ $\left(\mathbf{f}^{t}, \mathbf{g}^{t}\right)$ and $\mathcal{Y}=\mathbf{g}=\left[\begin{array}{ll}\mathbf{0} & \mathbf{I}\end{array}\right]^{\mathbf{t}} \mathcal{X}$. 


\section{Step II: Estimation of the High Resolution Image}

Once the hyperparameters have been estimated, the estimation of the high resolution image, $\mathbf{f}_{(\hat{\alpha}, \underline{\hat{\beta}})}$, is selected to minimize

$$
\hat{\alpha}\|\mathbf{C f}\|^{2}+\sum_{\mathbf{q}} \hat{\beta}_{\mathbf{q}}\left\|\mathbf{g}_{\mathbf{q}}-\mathbf{W}_{\mathbf{q}} \mathbf{f}\right\|^{2},
$$

which results in

$$
\mathbf{f}_{(\hat{\alpha}, \underline{\hat{\beta}})}=\mathbf{Q}(\hat{\alpha}, \underline{\hat{\beta}})^{-1} \sum_{\mathbf{q}} \hat{\beta}_{\mathbf{q}} \mathbf{W}_{\mathbf{q}}^{t} \mathbf{g}_{\mathbf{q}},
$$

where $\mathbf{Q}(\hat{\alpha}, \underline{\beta})=\hat{\alpha} \mathbf{C}^{t} \mathbf{C}+\sum_{\mathbf{q}} \hat{\beta}_{\mathbf{q}} \mathbf{W}_{\mathbf{q}}^{t} \mathbf{W}_{\mathbf{q}}$.

The estimation process we are using could be performed within the so called hierarchical Bayesian approach (see [6]) by including priors on the unknown parameters $\alpha$ and $\beta$. Note that the prior model in (9) plays an important role in the estimation of the high resolution image and the hyperparameters when an incomplete set $\left(\sum_{\mathbf{q}} \beta_{\mathbf{q}} \mathbf{W}_{\mathbf{q}}^{t} \mathbf{W}_{\mathbf{q}}\right.$ singular $)$ of low resolution images is used [5]. Note also that equation (12) can be solved using the method proposed in [6].

\section{Iterative Registration and Image Estimation}

Combining the methods proposed in section 3 to estimate the registration parameters, $\mathbf{d}$, and the algorithm to estimate the high resolution image, $\mathbf{f}$, described in section 4 , we can obtain an iterative procedure to estimate both of them.

Let $\mathbf{d}^{0}$ be the displacement vector obtained by applying the methods described in section 3 and $\mathbf{f}^{0}$ the image estimate obtained by the method described in section 4 when $\mathbf{d}=\mathbf{d}^{0}$. Then re-estimate the displacement vector $\mathbf{d}^{k}$ by using $\hat{\mathbf{u}}_{\mathbf{q}}$ obtained in section 3 and replacing $\hat{\mathbf{u}}$ (the initial estimate of the high resolution image obtained in section 3$)$, by $\mathbf{f}^{k-1}$. Recalculate $\mathbf{f}^{k}$ for this new displacement vector $\mathbf{d}^{k}$ using the method described in section 4 . The iterative procedure ends when $\left\|\mathbf{f}^{k}-\mathbf{f}^{k-1}\right\|^{2} /\left\|\mathbf{f}^{k-1}\right\|^{2}$ is less than a prescribed bound.

\section{Experimental Results}

A number of experiments were performed with the proposed algorithms over a set of images to evaluate their performance to estimate the high resolution image and registration parameters.

According to (1) the high resolution image in Fig. 1a, f, was first shifted to obtain a set of 16 high resolution images, $\mathbf{f}_{l 1, l 2}(x, y)=\mathbf{f}(x+l 1, y+l 2)$, $(x, y) \in\left\{0, \ldots, M_{1}\right\} \times\left\{0, \ldots, M_{2}\right\}, l 1, l 2=0, \ldots, 3$. Then each $\mathbf{f}_{l 1, l 2}$ was blurred using a motion blur of length 10 and downsampled by a factor of four. Gaussian noise was added to each low resolution image to obtain three sets of sixteen low resolution images, $\mathbf{g}_{l 1, l 2}$, with signal to noise ratio (SNR) equal to 20,30 and $40 \mathrm{~dB}$. 
Table 1. Estimated registration parameters for the low resolution image set with SNR of 20dB, by global correlation (first table) and local correlation (second table)

\begin{tabular}{|c|cccc|}
\hline \multicolumn{4}{|c|}{ GlobAl CORRELATION. } \\
Estimated Shifts in table \\
\hline \hline Real shifts & 0 & 1 & 2 & 3 \\
\hline 0 & $(0,0)$ & $(0,0)$ & $(0,0)$ & $(0,0)$ \\
1 & $(0,0)$ & $(0,0)$ & $(0,0)$ & $(0,0)$ \\
2 & $(0,0)$ & $(0,0)$ & $(0,0)$ & $(0,0)$ \\
3 & $(2,0)$ & $(1,0)$ & $(1,0)$ & $(1,0)$ \\
\hline
\end{tabular}

\begin{tabular}{|c|cccc|}
\hline \multicolumn{5}{|c|}{ LOCAL CORRELATION. } \\
Estimated SHIFTS In TABLE \\
\hline \hline Real shifts & 0 & 1 & 2 & 3 \\
\hline 0 & $(0,0)$ & $(0,1)$ & $(0,2)$ & $(0,3)$ \\
1 & $(1,0)$ & $(1,1)$ & $(1,2)$ & $(1,3)$ \\
2 & $(2,0)$ & $(2,1)$ & $(2,2)$ & $(2,3)$ \\
3 & $(3,0)$ & $(3,1)$ & $(3,2)$ & $(3,3)$ \\
\hline
\end{tabular}

Table 2. Estimated registration parameters for the low resolution image set with SNR of $30 \mathrm{~dB}$, by global correlation (first table) and local correlation (second table)

\begin{tabular}{|c|cccc|}
\hline \multicolumn{5}{|c|}{ GLOBAL CORRELATION. } \\
Estimated SHifts In table \\
\hline \hline Real shifts & 0 & 1 & 2 & 3 \\
\hline 0 & $(0,0)$ & $(0,0)$ & $(0,0)$ & $(0,0)$ \\
1 & $(0,0)$ & $(0,0)$ & $(0,0)$ & $(0,0)$ \\
2 & $(0,0)$ & $(0,0)$ & $(0,0)$ & $(0,0)$ \\
3 & $(1,0)$ & $(1,0)$ & $(1,0)$ & $(1,0)$ \\
\hline
\end{tabular}

\begin{tabular}{|c|cccc|}
\hline \multicolumn{5}{|c|}{ LOCAL CORRELATION. } \\
EstimATED SHIFTS IN TABLE \\
\hline \hline Real shifts & 0 & 1 & 2 & 3 \\
\hline 0 & $(0,0)$ & $(0,1)$ & $(0,2)$ & $(0,3)$ \\
1 & $(1,0)$ & $(1,1)$ & $(1,2)$ & $(1,3)$ \\
2 & $(2,0)$ & $(2,1)$ & $(2,2)$ & $(2,3)$ \\
3 & $(3,0)$ & $(3,1)$ & $(3,2)$ & $(3,3)$ \\
\hline
\end{tabular}

In order to test the performance of the proposed algorithms we ran the registration algorithms in section 3 and the reconstruction method described in section 4 on different sets of $\mathbf{q}$ randomly chosen low resolution images with $1 \leq \mathbf{q} \leq 16$. For comparison purposes, Fig. 1b depicts the zero-order hold upsampled image of $\mathbf{g}_{0,0}$ for $30 \mathrm{~dB}$ SNR $(\mathrm{PSNR}=13.68 \mathrm{~dB})$ while the bilinear interpolation of $\mathbf{g}_{0,0}$ is shown Fig. 1c $(\mathrm{PSNR}=14.22 \mathrm{~dB})$.

Tables 1 and 2 show, at convergence of the method described in section 5 , the estimated displacement vectors obtained using the methods described in sections 3.1 and 3.2 for the $20 \mathrm{~dB}$ and $30 \mathrm{~dB}$ cases, respectively. The estimated registration parameters did not change between the first and second iteration of the method.

The estimated high resolution image for the $30 \mathrm{~dB}$ case using local correlation and 6 low resolution images is depicted in Fig. 1d $(\mathrm{PSNR}=21.03 \mathrm{~dB})$.

\section{Conclusions}

In this paper we have presented an iterative method to estimate the registration parameters and reconstruct a high resolution image from a set of shifted low resolution observation. The method has been tested on synthetic images and has provided good estimates of both the high resolution image and displacement vectors.

The proposed method works for the case of shift displacements between the high resolution images. Extension to homographies, of particular interest for plane surfaces like registration plates or text, is under study. 


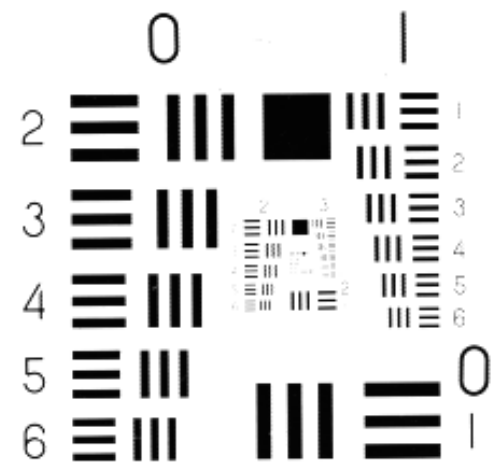

(a)

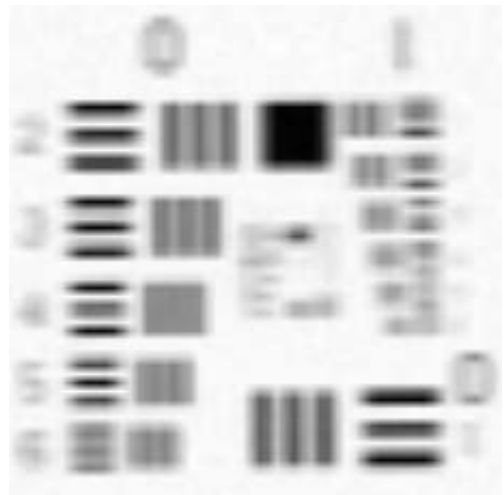

(c)

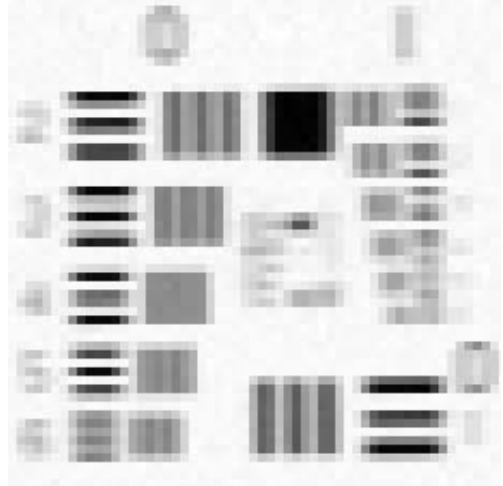

(b)

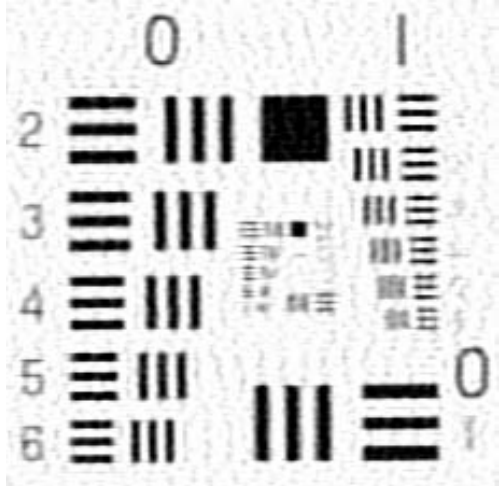

(d)

Fig. 1. (a) original image (b) zero order hold, (c) bilinear interpolation, (d) image obtained with the proposed method using 6 low resolution images

\section{References}

1. K. Aizawa, T. Komatsu, and T. Saito. A scheme for acquiring very high resolution images using multiple cameras. In IEEE Conference on Audio, Speech and Signal Processing, volume 3, pages 289-292, 1992.

2. C. M. Bishop, A. Blake, and B. Marthi. Super-resolution enhancement of video. In C. M. Bishop and B. Frey (Eds.), Proceedings Artificial Intelligence and Statistics, 2003.

3. C.J. Harris and M. Stephens. A combined corner and edge detector. In Proceedings 4th Alvey Vision Conference, pages 147-151, 1988.

4. M. G. Kang and S. Chaudhuri, editors. Super-resolution image reconstruction. IEEE Signal Processing Magazine, vol. 20, no. 3, 2003.

5. J. Mateos, M. Vega, R. Molina, and A.K. Katsaggelos. Bayesian image estimation from an incomplete set of blurred, undersampled low resolution images. In 1st Iberian Conference on Pattern Recognition and Image Analysis (IbPRIA2003), LNCS 2652, pages 445-452, 2003. 
6. R. Molina, M. Vega, J. Abad, and A.K. Katsaggelos. Parameter estimation in bayesian high-resolution image reconstruction with multisensors. IEEE Trans. on Image Processing, 12:1655-1667, 2003.

7. B.D. Ripley. Spatial Statistics. John Wiley, New York, 1981.

8. H. Stark and P. Oskoui. High-resolution image recovery from image-plane arrays, using convex projections. Journal of the Optical Society A, 6(11):1715-1726, 1989.

9. B. C. Tom, N. P. Galatsanos, and A. K. Katsaggelos. Reconstruction of a high resolution image from multiple low resolution images. In S. Chaudhuri, editor, Super-Resolution Imaging, chapter 4, pages 73-105. Kluwer Academic Publishers, 2001.

10. B. C. Tom and A. K. Katsaggelos. Reconstruction of a high-resolution image by simultaneous registration, restoration, and interpolation of low-resolution images". In Proceedings of the IEEE International Conference on Image Processing, volume 2, pages 539-542, 1995.

11. M. Vega, J. Mateos, R. Molina, and A.K. Katsaggelos. Bayesian parameter estimation in image reconstruction from subsampled blurred observations. In IEEE International Conference on Image Processing (ICIP-2003), volume II, pages 969973, 2003.

12. N.A. Woods, N.P. Galatsanos, and A.K. Katsaggelos. Em-based simultaneous registration, restoration, and interpolation of super resolved images. In Proceedings of the IEEE International Conference on Image Processing, volume II, pages 303306, 2003. 